\title{
Key Issues and Progress in Understanding Climate Risk in Africa
}

\author{
Katharine Vincent and Declan Conway
}

Abstract Adaptations and strategies to build resilience are needed to manage current impacts and will be increasingly vital as the world continues to warm. But making adaptation decisions can be complex, requiring careful consideration of multiple factors and perspectives, and balancing different priorities over different timescales. Society is embarking on a learning process that will continue for decades. This chapter and the book it introduces aim to contribute to this process. The book draws extensively from the Future Climate for Africa (FCFA) research programme that aimed to support adaptation and resilience in sub-Saharan Africa. In this chapter, we first briefly review the planning landscape for adaptation and building resilience and then consider how applications are changing the nature of climate information and the context of its use. This is followed

K. Vincent

Kulima Integrated Development Solutions, Pietermaritzburg, South Africa e-mail: katharine@kulima.com

D. Conway $(\bowtie)$

Grantham Research Institute on Climate Change and the Environment, London School of Economics and Political Science, London, UK e-mail: d.conway@lse.ac.uk

(C) The Author(s) 2021

D. Conway, K. Vincent (eds.), Climate Risk in Africa, https://doi.org/10.1007/978-3-030-61160-6_1 
by a review of the current status of climate information, particularly future projections for Africa and the enduring challenge that uncertainty represents to their active use. We then ask how we can improve the use of climate information for resilience building and adaptation and present an overview of the coming chapters. The demand for information and guidance on adaptation is continuing to grow, and is highlighting the need for new types and formats of data, and more innovative interactions with users to increase usability and application. Climate plays a dynamic role within complex, rapidly evolving social-ecological systems; this requires the climate science, resilience and adaptation communities to engage widely with other sectors and actors to make the agenda relevant and tractable for policy and practice.

Keywords Future Climate for Africa $\bullet$ Adaptation $\bullet$ Building resilience

\section{INTRODUCTION}

Our climate is changing-with major consequences for ecosystems and society. Adaptations and strategies to build resilience are needed to manage current impacts and will be increasingly vital as the world continues to warm. But making adaptation decisions can be complex, requiring careful consideration of multiple factors and perspectives, and balancing different priorities over different timescales. In particular, the fact that many adaptation benefits will accrue more acutely in the future means that they are often deprioritised relative to more immediate development challenges, particularly in Africa. This occurs against a context of uncertainty around the specific ways in which climate change will manifest at the local scale. Societies are only at the start of a learning process that will continue for decades.

This book aims to contribute to this process by developing our understanding of climate risk and its implications for approaches to adaptation and building resilience in Africa. We draw heavily on experiences from Future Climate for Africa (FCFA, https://futureclimateafrica.org/), an applied research programme that aimed to support adaptation and resilience in sub-Saharan Africa through better understanding of climate risk and promotion of climate information use to inform planning decisions over the medium (5-40 years) term future. Projects under this programme 
worked to improve the availability, accessibility and use of climate information in different decision-making contexts-from cities to water infrastructure to agriculture-in a range of countries. The book presents learning and experiences from this programme, focusing specifically on what does and does not work and why. In doing so, we critically reflect on a selection of trans-disciplinary approaches that bring together researchers and decision-makers to manage climate risk in the context of complex multi-dimensional problems. Our aim is that insights from these experiences can inform resilience building and adaptation across subSaharan Africa.

This chapter sets the scene by briefly reviewing the planning landscape for adaptation and building resilience in the following section. Afterwards, we consider how the range of potential uses of climate information is changing the nature of information that is produced, and then assess the current status of climate information, particularly future projections for Africa. Following that, we ask how we can improve the use of climate information for resilience building and adaptation, and then the final section provides an overview of the coming chapters.

\section{Planning for Adaptation and Building Resilience}

Recognition of the need for adaptation and building resilience has grown concurrently with awareness of climate change and the policy instruments that are in place to address it. The United Nations Framework Convention on Climate Change (UNFCCC) is the global policy arena for managing climate change. The framework convention addresses mitigation of the causes of climate change, and adaptation to the consequences of those changes. The latest legal instrument under the UNFCCC, the Paris Agreement, defines a Global Goal on Adaptation (Article 7).

The Global Goal on Adaptation aims to enhance adaptive capacity and resilience and to reduce vulnerability, with a view to contributing to sustainable development, and particularly ensuring that adaptation is adequate in light of the goal of limiting global warming to $2{ }^{\circ} \mathrm{C}$ (and pursuing efforts to limit it to below $1.5^{\circ} \mathrm{C}$ ). To ensure that this takes place, each Party to the UNFCCC is obliged to plan for adaptation (e.g. with a National Adaptation Plan) and communicate progress in those plans and their implementation through Adaptation Communications. Progress towards the Global Goal on Adaptation will be monitored every five years through a global stocktake. This complements Nationally Determined 
Contributions (NDCs) under the Paris Agreement, whose primary aim is to outline mitigation commitments but may also contain adaptation priorities. It also builds on National Adaptation Programmes of Action (NAPAs), which were submitted by Least Developed Country (LDC) Parties to outline their most pressing adaptation needs and inform the direction of adaptation finance under the UNFCCC.

Commitments for adaptation at the international level have been reflected at the national level, with countries around the world putting in place policies, strategies and legislation to address the challenges of climate change (e.g. see Averchenkova et al. 2017). As well as promoting adaptation, there is recognition that planning processes need to take into account the potential risks posed by climate change to ensure that the intended benefits of plans remain sustainable in the face of these risks. Given the significant role of international aid in some cases, this means that not only national governments but also multilateral and bilateral donors need to ensure that their plans are taking into account future climate conditions.

National governments are not the only actors considering climate risk. Many donors are now screening for climate risk among their aid portfolios, but this is still piecemeal and rather ad hoc. In theory, the World Bank and African Development Bank, who are among the major investors in infrastructure projects, require that all projects are screened for climate risk, and that design modifications are instituted if required to sustain the intended benefits, before funding can be approved. There is also increasing commitment within the private sector to identify and address climate risk; however, these assessments are not done routinely and their rigour and outcomes are not easy to establish as the results are rarely published (e.g. for hydropower, Lumbroso et al. 2015) and often insufficient for investors (TCFD 2019).

Planning for adaptation and screening for climate risk generally requires information about future climate. Demand for climate information is thus growing, and raising questions about what types and how much information is necessary, how to engage with this demand, and how to develop methods to promote its effective use in ways suitable for the diversity of situations in sub-Saharan Africa. 


\section{Decisions and Planning Needs Are Changing the Nature of Climate Information That Is Required}

There has been a significant improvement over recent decades in scientific capacity to understand the climate system and model the details of future climate. However, this improved scientific capacity for generating forecasts and projections does not simply translate into the type of information that is required by decision-makers for planning (Conway 2011; Nissan et al. 2019). Instead, there is often a "usability gap" resulting from a mismatch in temporal and spatial scales of information, and the ways in which uncertainty is embodied, as well as whether demand is fully appreciated and how information is communicated (Lemos et al. 2012).

We can take two cases for illustration. When planning for the coming season, a small-scale farmer might want to know when the rains are likely to start, and how long they are likely to last. This will determine what to plant (either what crop, or what variety of a crop e.g. an early maturing or normal duration variety) and when to plant it in order to ensure maximum production. When planning a water storage and distribution system to ensure availability for a growing urban population, a government ministry will want to know where it should place a dam and the associated infrastructure, and what their design should look like (e.g. in terms of dam capacity) in order to ensure maximum efficiency and reduce the risk of losses or excessive maintenance and repair costs due to floods and drought. Although there is scientific capacity to generate information to inform these decisions, it rarely matches the decision-makers' desired accuracy, format and presentation. Across Africa, the development and dissemination of seasonal climate forecasts has long been a particularly active area of climate research and applications, with important lessons for addressing the usability gap (Hansen et al. 2011). However, the nature of seasonal forecasts means that they are not always easy to interpret and use.

There are several reasons for a mismatch in information supply and demand with seasonal forecasts. Seasonal forecasts are probabilistic rather than deterministic, meaning that they provide the likelihood that the total volume of rainfall in a season will be above normal, normal and below normal. This poses several challenges for decision-making. Firstly, the rainfall patterns in sub-Saharan Africa are variable over time, and thus the medium- or long-term average that represents the 'normal' volume of seasonal rainfall against which the forecast for the coming season is compared can disguise significant variability. Comparing a coming season with 
an average is thus often difficult to visualise. Secondly, the probabilistic nature of the forecast is difficult to interpret. Dividing up 100\% into three probabilistic terciles often results in negligible differences-for example, a forecast might say there is $40 \%$ likelihood of above normal rainfall, $30 \%$ likelihood of normal rainfall, and $30 \%$ likelihood of below normal rainfall. The limited difference between the three categories means that it does not often give farmers usable information on what to expect. Thirdly, the spatial scale for the seasonal forecast is often large. Regional Climate Outlook Fora develop collectively agreed (consensus-based) seasonal forecasts at regional level, which are then contextualised by countries and sometimes downscaled to sub-national level. However, the large areas covered by seasonal forecasts are unlikely to have uniform conditions, which reduces the likelihood that the information will be accurate at high spatial resolution. Fourthly, seasonal forecasts focus on the total amount of rainfall that is likely to fall within a season (the variable for which forecast skill is most accurate), when it is the distribution of the rainfall that matters the most for planting decisions (which generally has low forecast skill).

Similar challenges of mismatch between supply and demand are evident for longer-term climate projections. Global Climate Models (GCMs) project future climates over the long term, typically until 2100 and beyond, which is longer than the timeframe of most planning decisions. The average lifespan of a dam, for example, is around 50 years, so the priority would be to know the future climate until around 2070. Typically the spatial resolution of GCMs has been coarse, with grid cells of hundreds of kilometres squared (although this has reduced over time). One of the biggest challenges with climate projections is that they embody multiple sources of uncertainty. Indeed, modelling anything into the future is subject to uncertainty. Future climate will depend on the concentrations of greenhouse gases in the atmosphere which depend on the evolution of human activities and any policy decisions to limit emissions. There is then uncertainty in how the multi-faceted components of the climate system will respond to those concentrations, and how they will interact with each other. Each of the over 60 GCMs in the world will capture these processes differently-adding an additional element of uncertainty. Unfortunately, uncertainty tends to increase at finer spatial and temporal scales, which is a problem because it is often finer-scale information that stakeholders request and which leads to the most significant risk (i.e. from extreme floods and droughts). More information on the background, use and 
presentation of GCM projections can be found in FCFA guides (e.g. FCFA 2016; Conway et al. 2017).

As a result of these uncertainties, there is often disagreement between models. While all models project warming, there is considerable divergence in rainfall conditions, with the model range including wetting and drying in most of Africa. This uncertainty makes it difficult for planners to know what they need to plan for. Added to this, the ways in which scientists visualise the outputs of GCMs with different plots is not always easy for non-specialists to understand and interpret (Fig. 1.1).

\section{Status of Climate Model Projections}

While there is clearly a need to better match climate information supply with the demands of users, there has been significant progress in the availability and quality of information over time. Temperature remains easier to project with confidence than rainfall, which is subject to the interaction of a wider range of factors acting at the local level. One of the first reviews was published 25 years ago (Hulme 1994), showing GCM results with a mid-range greenhouse gas emission scenario for 2050 that projected warming of most of tropical Africa by less than $1.2^{\circ} \mathrm{C}$ (from 1990). Rainfall was projected to increase over Africa, except for the northern third where drier conditions were projected, although with high uncertainty, particularly over the Sahel. This situation prompted the observation that 'This wide range of possible precipitation changes for Africa makes it problematic to develop sensible response strategies to greenhouse gas induced climate change in Africa' (Hulme 1994, p. 39).

Given the different capacities of different models to represent and project the climate, best practice has always been to use collections of models, or "ensembles", and to consider the multi-model mean and the intermodel range. By 2001, with a sample of ten updated or new GCMs, Hulme et al. (2001) revised projections for Africa showing consistent patterns of warming from $2^{\circ}$ to $6^{\circ} \mathrm{C}$ by 2100 . Confidence in the magnitude and direction of change in regional rainfall was still low, leading the authors to suggest concentrating on vulnerability reduction and strengthening capacity to adapt to climate variability would bring immediate benefits and build capacity to adapt to longer-term changes in climate. However, broad spatial patterns appeared for the December to February season indicating wetting over East and central Africa and drying over parts of southern Africa. These results established spatial patterns of change that have 


\section{WHY ARE THERE SO MANY CLIMATE PROJECTIONS (GCM)} AND WAYS OF VISUALISING THEM?

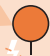

UNCERTAINTY IN HOW THE CLIMATE WILL CHANGE IN FUTURE NATURAL VARIABILITY | CÓMPLLEX CLIMATE'PROCESSES | RATE, Ó FUTURE EMISSIONS

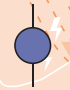

MANY SETS OF CLIMATE MODEL PROJECTIONS

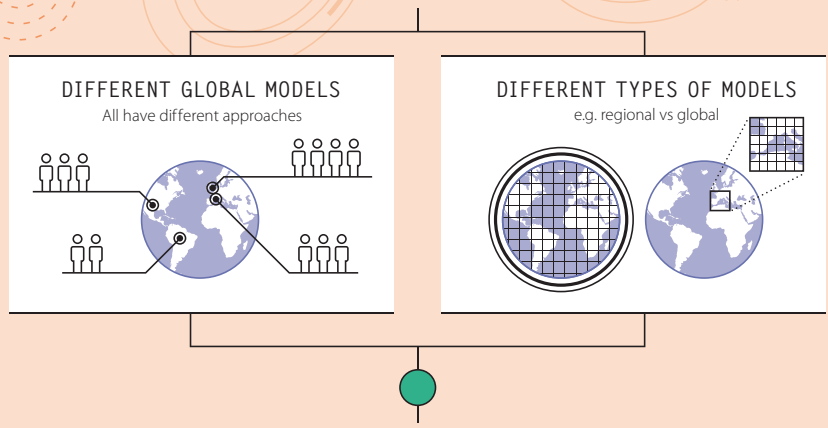

MANY WAYS OF VISUALISING THE PROJECTIONS

ALL HAVE PROS AND CONS

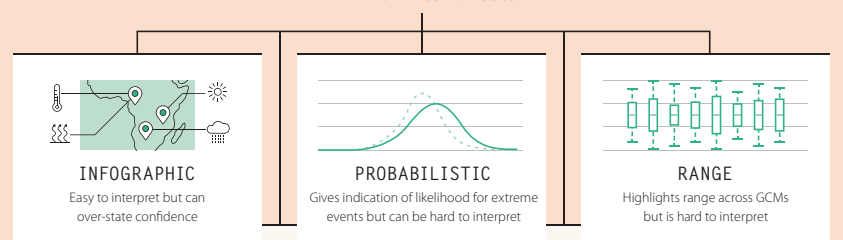

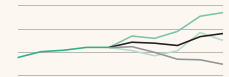

TIME SERIES

Highlights extremes and variability. can also include GCM range

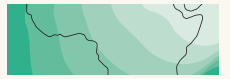

MAP

Shows spatial patterns of change can also include GCM range

Fig. 1.1 The various ways of presenting Global Climate Model outputs 
remained fairly stable throughout the subsequent multi-model assessments reported in the Fourth and Fifth Intergovernmental Panel on Climate Change (IPCC) reports (Coupled Model Intercomparison Project (CMIP) CMIP3, Christensen et al. 2007; CMIP5, Christensen et al. 2013).

However, similarities between model results do not necessarily indicate accuracy of projections. For example, there is a discrepancy between an observed drying trend in East African March to May rainfall and the projections for increasing rainfall in the future (the East African paradox; Rowell et al. 2015). For the Sahel, the observed multi-decadal variability is a crucial test for GCMs, and while this is simulated by many, they do not capture the scale of observed oscillations at multi-decadal timescales (Biasutti 2013). For southern Africa, projections of drying in early summer are robust, but extreme drying simulated by some models appears unlikely because these models simulate too much rainfall in the present climate (Munday and Washington 2018). Some approaches therefore consider constraining model selection by identifying those that most accurately represent the climate of a particular region or the mechanisms by which it changes. But, constraining models in this way is contentious (it requires explicit value judgements) and may have a limited effect on the range of uncertainty, as found for East Africa (Rowell et al. 2016; Chap. 6).

\section{How Can We Improve the Use of Climate INFORMATION FOR AdAPTATION AND BUILDING RESILIENCE?}

There are various ways to overcome the usability gap and ensure that the improved availability of climate information translates into effective adaptation and resilience building. The important factors for successful information use can be broadly categorised as credibility (perceived technical quality of information), legitimacy/trust (belief that the information seeks to serve the users' interests) and salience (relevance to users' needs) (Cash et al. 2003). Another categorisation uses fit, interplay and interaction (Lemos et al. 2012). Fit includes users' perceptions of how climate information fits with the organisational context; interplay considers how well information can integrate with pre-existing knowledge or information in the organisation; and interaction deals with the relationship between the information producers and the users (Soares and Dessai 2016). Development and dissemination of seasonal climate forecasts has been a 
particularly active area of climate research and applications in Africa. The importance of framing any new information in the context of existing risk management practices is a key lesson from this work.

As outlined earlier, uncertainty-how to characterise it and how to deal with it - has been and continues to be a defining feature of research and practice on adaptation. The wide spread in model results over much of Africa has remained a stubborn feature since the earliest days of multimodel comparisons and has implications for the credibility of information. The key role that rainfall plays in livelihood systems across Africa makes this particularly challenging for decision-making. There are limits to the accuracy of projections that can be obtained about the future, which will always be subject to some uncertainty. This calls into question the topdown, supply-driven "predict then act" approach, whereby climate projections determine risk, and then adaptation options are identified to respond to that risk.

Turning this approach on its head, a variety of decision-driven approaches have arisen that look at the planning decision that needs to be made and ensure that the decision will be resilient in the context of a range of potential futures (thereby addressing the uncertainty of any future projections). One branch is known as Robust Decision-Making (RDM) or Decision-Making Under Uncertainty (DMUU). These approaches recognise that planning decisions with long-term lifespans need to be made, with deep uncertainties that cannot be reduced by gathering more information, but can be addressed by moving from predict-then-act approaches to assess-risk-of-policy approaches (Lempert et al. 2006). RDM methods can involve a combination of top-down and bottom-up approaches in which modelling methods are informed by stakeholder consultation processes. Presenting information about future risks and uncertainty can be approached in different ways, such as through narratives or storylines that combine process-based understanding of physical climate communicated in a bespoke manner, within hypothetical settings or using expert judgement techniques (e.g. Chap. 2).

On shorter timescales some humanitarian and development agencies are developing decision protocols that use forecast information for advance release of finance or other types of early action for disaster risk management (Forecast-based Action, FbA; Wilkinson et al. 2018). Examples of $\mathrm{FbA}$ use a variety of financing tools, including dedicated funds, specially allocated funds in emergency response funds, insurance and direct links to regular resource allocation processes. FbA programmes have been 
deployed through various delivery mechanisms, including social protection systems. There has also been a move in this field to frame actions as 'no regrets' or 'low regrets' which are likely to result in humanitarian or wider development benefits irrespective of how the situation plays out, especially for seasonal timescales given high levels of uncertainty (Wilkinson et al. 2018).

Legitimacy issues such as defining aims, involvement in processes and ownership of outcomes are critical to agendas promoting climate information use. This includes engaging in climate science and the development of GCM projections or being able to tailor them to their national contexts. Increasing availability of projection-generating toolkits has contributed to $90 \%$ of 189 countries including climate projections in their vulnerability and adaptation assessments that form part of their National Communications to the UNFCCC (Skelton et al. 2019). However, the wide adoption of GCM projections obscures major differences in capacity to generate and customise global climate science to national/local context: capacity is strongly skewed in favour of countries in the global North (Haunschild et al. 2016). Infrastructure and capacity gaps and lack of funding are known to be important for many National Meteorological and Hydrological Services (NMHS), and while recent extensive funding and initiatives are going some way to address these concerns, political and economic considerations require careful attention (Harvey et al. 2019). These issues feed into broader research on the political dimensions of adaptation through which ideas, power and resources are determined by different groups across scales ranging from the global North and South to between and within communities (Tanner and Allouche 2011; Eriksen et al. 2015). Other concerns include debates about the role of different actors in the process. For example, some warn that commercialised models of climate service provision might exacerbate the challenge of using information from climate science to inform adaptation by gatekeeping access on the basis of ability to pay, which is particularly an issue in Africa (Webber and Donner 2017).

To achieve salience, there is a need to translate model results into userrelevant information that is contextualised to suit the specific needs of agencies, communities and individuals. This often requires a role for intermediaries (Dilling and Lemos 2011). Limits to the spatial detail of GCM projections and a common focus on timescales far into the future are significant challenges to this goal. A prerequisite is to understand decisionmaking contexts and information needs for potential "users" of such 
climate information (Carr et al. 2020; Harvey et al. 2019). Bringing together the "producers" and "users" of climate information can help promote the dialogue required for each to understand the other's perspectives, abilities and needs, bearing in mind that limited resources and skillsets may be important barriers (e.g. Chap. 3). These various actors working together in a process of co-production can improve the likelihood that credible, legitimate and salient information is produced, which increases the likelihood of application (Carter et al. 2019). However, thus far coproduction of climate services is still in its infancy, especially in Africa, and there is a need for more rigorous evaluation of its utility (Wall et al. 2017).

\section{Conclusion and Outline of Following Chapters}

The need to adapt and build resilience is clear. The demand for information and guidance to support this process is continuing to grow, and is highlighting the need for new types and formats of information, and more innovative interactions with users to increase usability and use (Vincent et al. 2020). Progress towards effective linkage between top-down, supplyside approaches that aim to address the availability of information with bottom-up, demand-side approaches where information is defined by decision contexts has been slow. This book addresses this gap through real-world examples that apply novel approaches to knowledge creation.

The following chapters provide an expanded context, informed by practice, to climate research in Africa, recognising the important relevance of shorter timescales but focusing on longer term (roughly 5-40 years) timescales for adaptation. In Chap. 2, Jack et al. introduce the concept of distillation and its relationship with climate information and definitions of reliability and robustness. They describe an example of information distillation using complementary approaches to GCM projections based on narratives. In Chap. 3, Vincent et al. reflect on the role of process-how activities are designed and undertaken-what principles should be considered (e.g. salience, credibility and legitimacy) and who is or should be involved. They consider what we are learning about a role for coproduction from practical attempts to employ it. In Chap. 4, Audia et al. argue for the need to add equity to the principles of flexibility, robustness and low economic regrets (FREE) that already characterise DMUU.

Chapters 5-8 then present case studies from Malawi, Tanzania, Zambia and Uganda-four countries where the FCFA programme has supported adaptation and resilience building through improving the provision of 
climate information that is useful and usable to decision-makers. In Chap. 5, Tembo-Nhlema et al. illustrate how Participatory Scenario Planning (PSP) has been used on seasonal forecasts in Malawi to generate useful and usable information for farmers. In Chap. 6, Siderius et al. present an approach for reducing uncertainty in GCM projections to inform decisions around water, energy and the environment in the Rufiji River basin of Tanzania and how such approaches benefit from user-defined performance indicators. In Chap. 7, Taylor et al. illustrate the process through which various sources of climate information were integrated into Lusaka's Strategic Plan for 2017-2021. In Chap. 8, Cornforth et al. reflect on ways to evaluate the impact that climate information has on decision-making through quantitatively assessing the status of livelihoods under different climate scenarios.

The case study chapters are guided by a series of questions designed to reflect the multidimensional nature of adaptation and some of the issues often encountered in practice:

- What are the characteristics of the decision problem and how are they defined and by whom?

- What kinds of interactions occur and who is involved in them?

- What are the key contextual factors, including the significance of historical climate risks and the role of institutions and governance?

- How are climate risks characterised and communicated, and over which timescales?

- To what extent does uncertainty about climate feature in the case study?

- To what extent are non-climate considerations important and how they are addressed?

- What are the reflections-what works well and why?

In Chap. 9, we reflect on the experiences outlined in the book noting that at their core are attempts to initiate and inform conversations about climate risk and the need for adaptation and resilience building. We consider these conversations and what they mean for the growing adaptation agenda. Africa is urbanising rapidly and is in the midst of major infrastructure expansion which is changing exposure and sensitivity to extremes, and generating new hazard combinations. The research presented in this book recognises that climate plays a dynamic role within complex environment-society processes. This requires adaptation researchers to 
engage with other sectors and actors to make the agenda relevant and tractable across policy and practice arenas.

\section{REFERENCES}

Averchenkova, A., Fankhauser, S., \& Nachmany, M. (Eds.). (2017). Trends in climate change legislation. London: Edward Elgar Publishing.

Biasutti, M. (2013). Forced Sahel rainfall trends in the CMIP5 archive. Journal of Geophysical Research: Atmospheres, 118(4), 1613-1623.

Carr, E. R., Goble, R., Rosko, H. M., Vaughan, C., \& Hansen, J. (2020). Identifying climate information services users and their needs in sub-Saharan Africa: A review and learning agenda. Climate and Development, 12(1), 23-41.

Carter, S., Steynor, A., Vincent, K., Visman, E., \& Waagsaether, K. (2019). Co-production of African weather and climate services. Manual. Cape Town: Future Climate for Africa and Weather and Climate Information Services for Africa. Retrieved from https://futureclimateafrica.org/coproduction-manual

Cash, D. W., Clark, W. C., Alcock, F., Dickson, N. M., Eckley, N., Guston, D. H., Jäger, J., \& Mitchell, R. B. (2003). Knowledge systems for sustainable development. Proceedings of the National Academy of Sciences, 100(14), 8086-8091. https://doi.org/10.1073/pnas.1231332100.

Christensen, J. H., et al. (2007). Regional climate projections. In S. Solomon et al. (Eds.), Climate change 2007: The physical science basis. Contribution of working group I to the fourth assessment report of the intergovernmental panel on climate change. Cambridge University Press.

Christensen, J. H., et al. (2013). Climate phenomena and their relevance for future regional climate change. In T. F. Stocker, et al. (Eds.), Climate change 2013: The physical science basis. Contribution of working group I to the fifth assessment report of the intergovernmental panel on climate change. Cambridge University Press.

Conway, D. (2011). Adapting climate research for development in Africa. Wiley Interdisciplinary Reviews: Climate Change, 2(3), 428-450.

Conway, D., Vincent, K., Grainger, S., Archer van Garderen, E., \& Pardoe, J. (2017). How to understand and interpret global climate model results. Cape Town: Future Climate For Africa. Retrieved from http://kulima.com/wpcontent/uploads/2017/10/FCFA_GCM-guide-web.pdf

Dilling, L., \& Lemos, M. C. (2011). Creating usable science: Opportunities and constraints for climate knowledge use and their implications for science policy. Global Environmental Change, 21(2), 680-689.

Eriksen, S. H., Nightingale, A. J., \& Eakin, H. (2015). Reframing adaptation: The political nature of climate change adaptation. Global Environmental Change, $35,523-533$. 
Future Climate for Africa (FCFA). (2016). Climate models: What they show us and how they can be used in planning. Cape Town: Future Climate for Africa. Retrieved from http://kulima.com/wp-content/uploads/2017/10/FCFA_ Climate_Models_WEB.pdf

Hansen, J. W., Mason, S. J., Sun, L., \& Tall, A. (2011). Review of seasonal climate forecasting for agriculture in sub-Saharan Africa. Experimental Agriculture, $47(2), 205-240$.

Harvey, B., Jones, L., Cochrane, L., \& Singh, R. (2019). The evolving landscape of climate services in sub-Saharan Africa: What roles have NGOs played? Climatic Change, 1-18.

Haunschild, R., Bornmann, L., \& Marx, W. (2016). Climate change research in view of bibliometrics. PLoS One, 11(7), e0160393.

Hulme, M. (1994). Regional climate change scenarios based on IPCC emissions projections with some illustrations for Africa. Area, 33-44.

Hulme, M., Doherty, R., Ngara, T., New, M., \& Lister, D. (2001). African climate change: 1900-2100. Climate Research, 17(2), 145-168.

Lemos, M. C., Kirchhoff, C. J., \& Ramprasad, V. (2012). Narrowing the climate information usability gap. Nature Climate Change, 2(11), 789-794. https:// doi.org/10.1038/nclimate1614.

Lempert, R. J., Groves, D. G., Popper, S. W., \& Bankes, S. C. (2006). A general, analytic method for generating robust strategies and narrative scenarios. Management Science, 52(4), 514-528.

Lumbroso, D. M., Woolhouse, G., \& Jones, L. (2015). A review of the consideration of climate change in the planning of hydropower schemes in sub-Saharan Africa. Climatic Change, 133(4), 621-633.

Munday, C., \& Washington, R. (2018). Systematic climate model rainfall biases over Southern Africa: Links to moisture circulation and topography. Journal of Climate, 31(18), 7533-7548.

Nissan, H., Goddard, L., de Perez, E. C., Furlow, J., Baethgen, W., Thomson, M. C., \& Mason, S. J. (2019). On the use and misuse of climate change projections in international development. Wiley Interdisciplinary Reviews: Climate Change, 10(3), e579.

Rowell, D. P., Booth, B. B., Nicholson, S. E., \& Good, P. (2015). Reconciling past and future rainfall trends over east Africa. Journal of Climate, 28(24), 9768-9788.

Rowell, D. P., Senior, C. A., Vellinga, M., \& Graham, R. J. (2016). Can climate projection uncertainty be constrained over Africa using metrics of contemporary performance? Climatic Change, 134(4), 621-633.

Skelton, M., Porter, J. J., Dessai, S., Bresch, D. N., \& Knutti, R. (2019). Customising global climate science for national adaptation: A case study of climate projections in UNFCCC's National Communications. Environmental Science \& Policy, 101, 16-23. 
Soares, M. B., \& Dessai, S. (2016). Barriers and enablers to the use of seasonal climate forecasts amongst organisations in Europe. Climatic Change, $137(1-2), 89-103$.

Tanner, T., \& Allouche, J. (2011). Towards a new political economy of climate change and development. Institute of Development Studies Bulletin, 42(3), 1-14. https://doi.org/10.1111/j.1759-5436.2011.00217.x

TCFD. (2019). Task force on climate-related financial disclosures: status report 2019. 144.

Vincent, K., Conway, D., Dougill, A. J., Pardoe, J., Archer, E., Bhave, A. G., Henriksson, R., Mittal, N., Mkwambisi, D., Rouhaud, E., \& Tembo-Nhlema, D. (2020). Re-balancing climate services to inform climate-resilient planning - A conceptual framework and illustrations from sub-Saharan Africa. Climate Risk Management, 29, 100242.

Wall, T. U., Meadow, A. M., \& Horganic, A. (2017). Developing evaluation indicators to improve the process of coproducing usable climate science. Weather Climate and Society, 9(1), 95-107. https://doi.org/10.1175/ WCAS-D-16-0008.1.

Webber, S., \& Donner, S. D. (2017). Climate service warnings: Cautions about commercializing climate science for adaptation in the developing world. Wiley Interdisciplinary Reviews: Climate Change, 8(1), e424.

Wilkinson, E., Weingärtner, L., Choularton, R., Bailey, M., Todd, M., Kniveton, D., \& Cabot-Venton, C. (2018). Forecasting disasters, averting hazards: Implementing forecast-based early action at scale. London: Overseas Development Institute.

Open Access This chapter is licensed under the terms of the Creative Commons Attribution 4.0 International License (http://creativecommons.org/licenses/ by $/ 4.0 /$ ), which permits use, sharing, adaptation, distribution and reproduction in any medium or format, as long as you give appropriate credit to the original author(s) and the source, provide a link to the Creative Commons licence and indicate if changes were made.

The images or other third party material in this chapter are included in the chapter's Creative Commons licence, unless indicated otherwise in a credit line to the material. If material is not included in the chapter's Creative Commons licence and your intended use is not permitted by statutory regulation or exceeds the permitted use, you will need to obtain permission directly from the copyright holder.

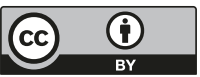

